\title{
Les déséquilibres entre les sexes dans la profession enseignante
}

- Historiquement, dans les pays de l'OCDE, la profession enseignante a toujours été à large dominante féminine. Le pourcentage de femmes dans le corps enseignant a augmenté ces dix dernières années, atteignant $68 \%$ en 2014, tous niveaux d'enseignement confondus.

- Le déséquilibre entre les sexes diminue progressivement avec l'élévation du niveau d'enseignement, les femmes représentant $97 \%$ du corps enseignant dans le préprimaire, contre $43 \%$ dans l'enseignement tertiaire. Entre 2005 et 2014, l'écart entre les sexes s'est creusé dans l'enseignement primaire et secondaire, mais s'est réduit dans l'enseignement tertiaire.

- Les hommes enseignant dans le primaire gagnent $71 \%$ des revenus d'autres actifs occupés du même sexe diplômés de l'enseignement tertiaire ; ce pourcentage des revenus de référence passe à $81 \%$ dans le deuxième cycle de l'enseignement secondaire. En revanche, quel que soit le niveau auquel elles enseignent, les femmes gagnent au moins $90 \%$ des revenus d'autres actives occupées diplômées de l'enseignement tertiaire. L'ampleur de cette différence de revenus relatifs peut contribuer à rendre la profession enseignante plus attrayante aux yeux des femmes.

- C'est dans la nouvelle génération d'enseignants (âgés de moins de 30 ans) que le pourcentage de femmes est le plus élevé, ce qui suscite des inquiétudes concernant l'accentuation des déséquilibres entre les sexes au fil du temps - en particulier aux niveaux inférieurs d'enseignement, où les femmes sont largement majoritaires dans le corps enseignant.

La profession enseignante connaît un déséquilibre marqué - et croissant - entre les sexes

L'enseignement est une profession fortement liée au sexe : les femmes représentent plus de deux tiers du corps enseignant de l'enseignement préprimaire à l'enseignement tertiaire, en moyenne, dans les pays de I'OCDE. Ce déséquilibre est particulièrement marqué en Estonie, en Fédération de Russie, en Lettonie et en Lituanie, où environ quatre enseignants sur cinq sont des femmes. Parmi les 31 pays disposant de données, le Japon est le seul où les femmes sont moins nombreuses que les hommes dans le corps enseignant (elles y représentent $48 \%$ des effectifs, tous niveaux d'enseignement confondus). Ce constat surprenant peut en partie s'expliquer par la participation plus faible des Japonaises au marché du travail par rapport aux femmes d'autres pays (OCDE, 2012).

On constate une "féminisation » progressive de la profession enseignante ces dix dernières années. En moyenne, dans les pays de I'OCDE, le pourcentage de femmes dans le corps enseignant est passé de $61 \%$ en 2005 à $65 \%$ en 2010, pour atteindre 68 \% en 2014. Cette tendance à la hausse s'observe dans tous les pays disposant de données, quel que soit leur pourcentage de femmes dans le corps enseignant en 2005 (graphique 1). L'ampleur de cette augmentation varie toutefois sensiblement entre les pays, avec une hausse de moins de $1 \%$ aux États-Unis et en Slovénie, mais de plus de $15 \%$ en Allemagne, en Grèce et au Japon.

\section{Graphique 1. Pourcentage de femmes dans le corps enseignant,} tous niveaux d'enseignement confondus $(2005,2014)$

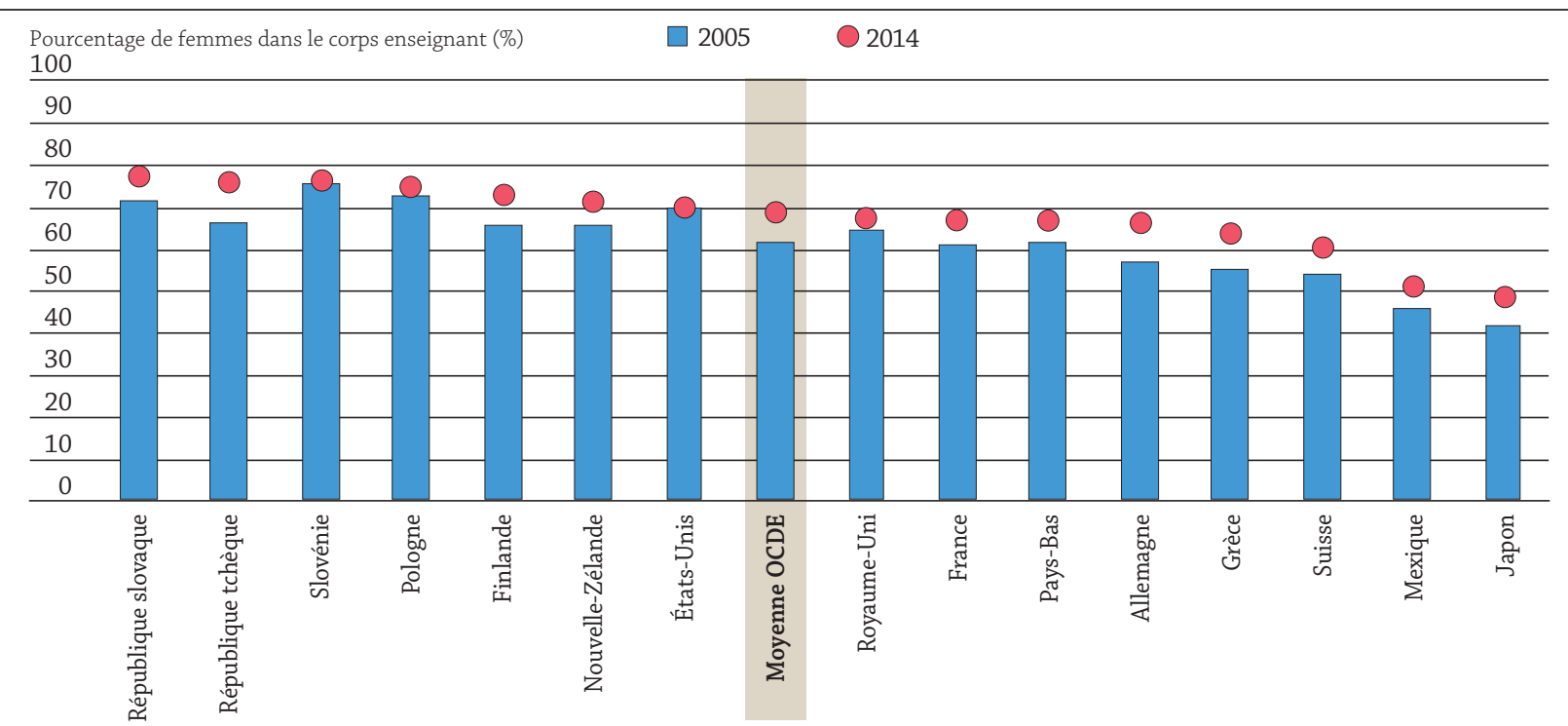

Remarque : Seuls sont inclus les pays disposant de données pour les deux années de référence. Pour de plus amples informations, consulter l'annexe 3 (www.oecd.org/fr/education/regards-sur-l-education-19991495.htm).

Les pays sont classés par ordre décroissant du pourcentage de femmes dans le corps enseignant en 2014

Source : OCDE (2016), Regards sur l'éducation (base de données). 
L'écart entre les sexes varie toutefois sensiblement selon le niveau de responsabilité, la discipline et le niveau d'enseignement

Si les femmes sont majoritaires dans la profession enseignante, elles sont toutefois relativement sous-représentées aux postes de direction. En moyenne, dans les pays de l'OCDE, les femmes représentent $68 \%$ du corps enseignant dans le premier cycle du secondaire, mais seulement $45 \%$ des chefs d'établissement (OCDE, 2016). Ce constat est particulièrement frappant quand l'on sait que les chefs d'établissement sont en général recrutés dans le corps enseignant - ce qui semble indiquer que les enseignantes sont moins susceptibles d'être promues à la fonction de chef d'établissement que leurs collègues de sexe masculin.

Dans le corps enseignant lui-même, des déséquilibres s'observent entre les sexes selon la discipline enseignée. Dans le premier cycle du secondaire, les femmes sont moins nombreuses parmi les professeurs de sciences, de mathématiques et de technologie que dans l'ensemble du corps enseignant (OCDE, 2015a). Le pourcentage de femmes dans le corps enseignant diminue en outre sensiblement avec l'élévation du niveau d'enseignement. En moyenne, dans les pays de l'OCDE, les femmes représentent ainsi $97 \%$ du corps enseignant dans le préprimaire, $82 \%$ dans le primaire, mais $63 \%$ dans le secondaire et $43 \%$ dans l'enseignement tertiaire. Ce constat vaut pour la plupart des pays disposant de données. Dans le primaire, les femmes représentent plus de $60 \%$ du corps enseignant dans tous les pays sauf en Arabie saoudite et en Turquie. En revanche, dans l'enseignement tertiaire, les femmes ne sont plus nombreuses que les hommes dans le corps enseignant que dans quatre pays : en Fédération de Russie, en Finlande, en Lettonie et en Lituanie (graphique 2).

\section{Graphique 2. Pourcentage de femmes dans le corps enseignant, selon le niveau d'enseignement (2014)}

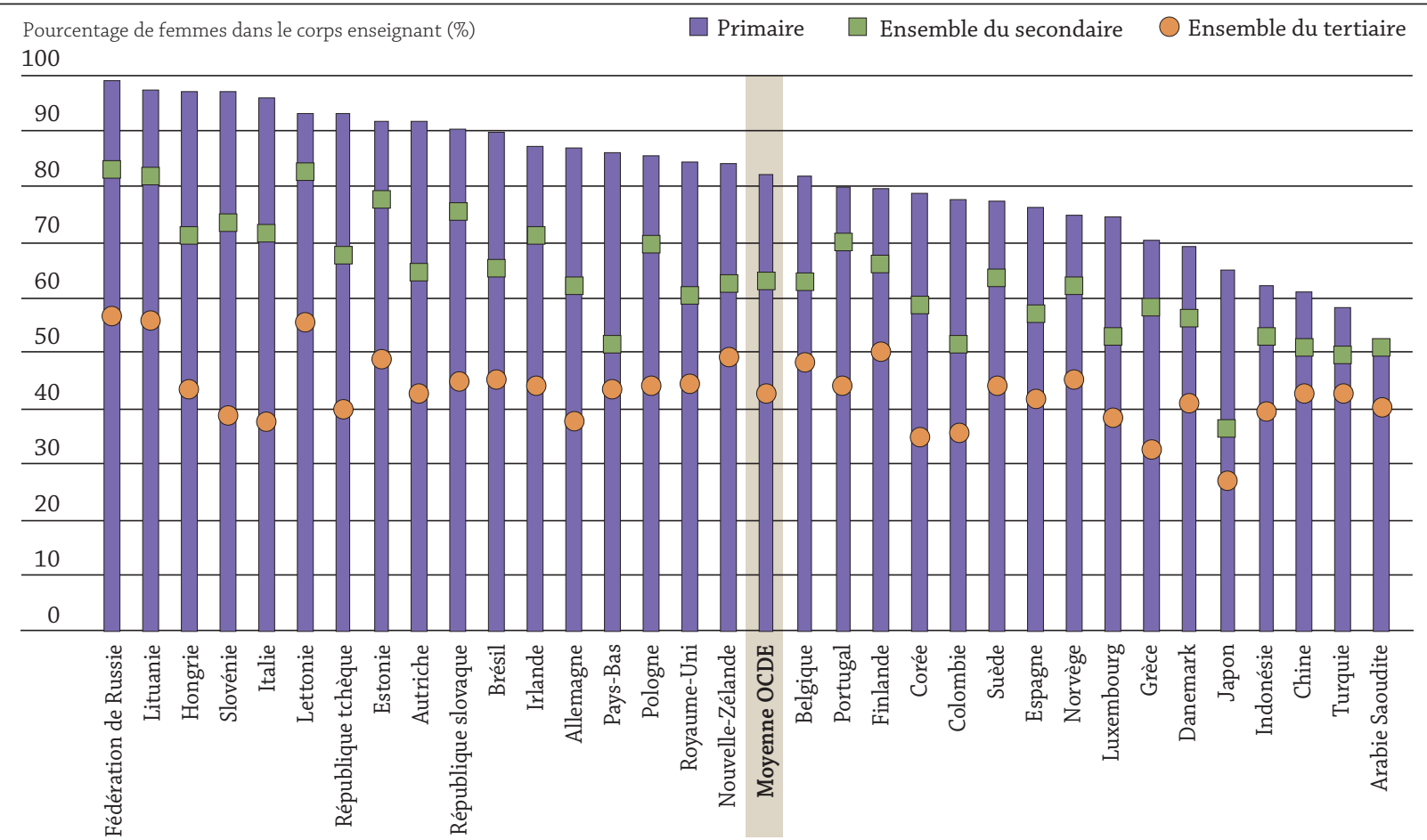

Remarque : Seuls sont inclus les pays disposant de données pour l'ensemble des catégories. Pour de plus amples informations, consulter l'annexe 3 de l'édition 2016 de Regards sur l'éducation (www.oecd.org/fr/education/regards-sur-l-education-19991495.htm).

Les pays sont classés par ordre décroissant du pourcentage de femmes dans le corps enseignant du primaire.

Source : OCDE (2016), Regards sur l'éducation 2016 : Les indicateurs de l'OCDE, Éditions OCDE, Paris, http://dx.doi.org/10.1787/eag-2016-fr, tableau D5.3.

\section{Comment expliquer ce déséquilibre entre les sexes dans la profession enseignante?}

Entre 2005 et 2014, le taux d'emploi des femmes est passé de $56 \%$ à $59 \%$, en moyenne, dans les pays de l'OCDE. Bien que cette relation puisse ne pas s'appliquer à tous les pays, la hausse de la participation des femmes au marché du travail est susceptible d'avoir contribué à la « féminisation » de la profession enseignante. Ainsi, en Allemagne et au Japon - qui, avec la Grèce, comptent parmi les trois pays1 présentant la plus forte augmentation du pourcentage de femmes dans le corps enseignant au cours des dix dernières années -, le taux d'emploi des femmes a enregistré une hausse de plus de $11 \%$, contre les $8 \%$ observés en moyenne dans les pays disposant de données. En outre, les stéréotypes selon lesquels hommes et femmes excelleraient dans des domaines différents et seraient plus aptes à exercer certaines professions sont susceptibles d'entraîner une certaine forme de ségrégation entre les sexes dans différents domaines de l'éducation. Ainsi, le faible pourcentage de femmes parmi les professeurs de sciences pourrait résulter de la représentation sociale des sciences comme une discipline masculine - préjugé qui dissuaderait les femmes d'entreprendre des études tertiaires dans ce domaine (OCDE, 2015b).

Des considérations d'ordre économique sont également susceptibles d'avoir une incidence sur le parcours professionnel des hommes et des femmes au sein de la profession enseignante, posant le problème d'éventuels déséquilibres salariaux entre les sexes dans les différents niveaux d'enseignement. À ce sujet, il est intéressant de noter qu'alors que le pourcentage de femmes dans le corps enseignant tend 1. Sur les 15 pays disposant de données à la fois pour 2005 et 2014 (voir le graphique 1). 


\section{INDICATEURS DE L'ÉDUCATION À LA LOUPE}

à díminuer avec l'élévation du niveau d'enseignement, les salaires, eux, tendent à augmenter. Ainsi, dans les pays de l'OCDE, le salaire effectif moyen d'un enseignant âgé de 25 à 64 ans augmente progressivement avec l'élévation du niveau d'enseignement, passant de 37300 USD dans le préprimaire à 46600 USD dans le deuxième cycle du secondaire. En moyenne, les femmes gagnent légèrement moins que les hommes dans le primaire et le secondaire, même si l'écart salarial entre les sexes ne dépasse jamais $3 \%$.

Les différences entre les sexes sont plus significatives lorsque la comparaison porte sur le salaire des enseignants par rapport aux revenus d'autres actifs occupés diplômés de l'enseignement tertiaire. En moyenne, dans les pays de l'OCDE, les hommes (âgés de 25 à 64 ans) enseignant dans le primaire gagnent $71 \%$ des revenus d'autres actifs occupés du même sexe diplômés de l'enseignement tertiaire. Ce pourcentage des revenus de référence passe à $76 \%$ dans le premier cycle du secondaire et à $81 \%$ dans le deuxième cycle du secondaire. Les enseignantes ont des revenus relatifs significativement plus élevés. Les femmes enseignant dans le primaire gagnent ainsi plus de $90 \%$ des revenus d'autres actives occupées diplômées de l'enseignement tertiaire, et même légèrement plus que ces dernières lorsqu'elles enseignent dans les premier et deuxième cycles du secondaire (graphique 3). Ces écarts importants de revenus relatifs entre les hommes et les femmes sont susceptibles d'avoir rendu la profession enseignante plus attrayante aux yeux des femmes, en particulier aux niveaux inférieurs d'enseignement.

\section{Graphique 3. Ratio entre le salaire des enseignants du primaire et les revenus d'autres actifs occupés diplômés de l'enseignement tertiaire, selon le sexe (2014)}

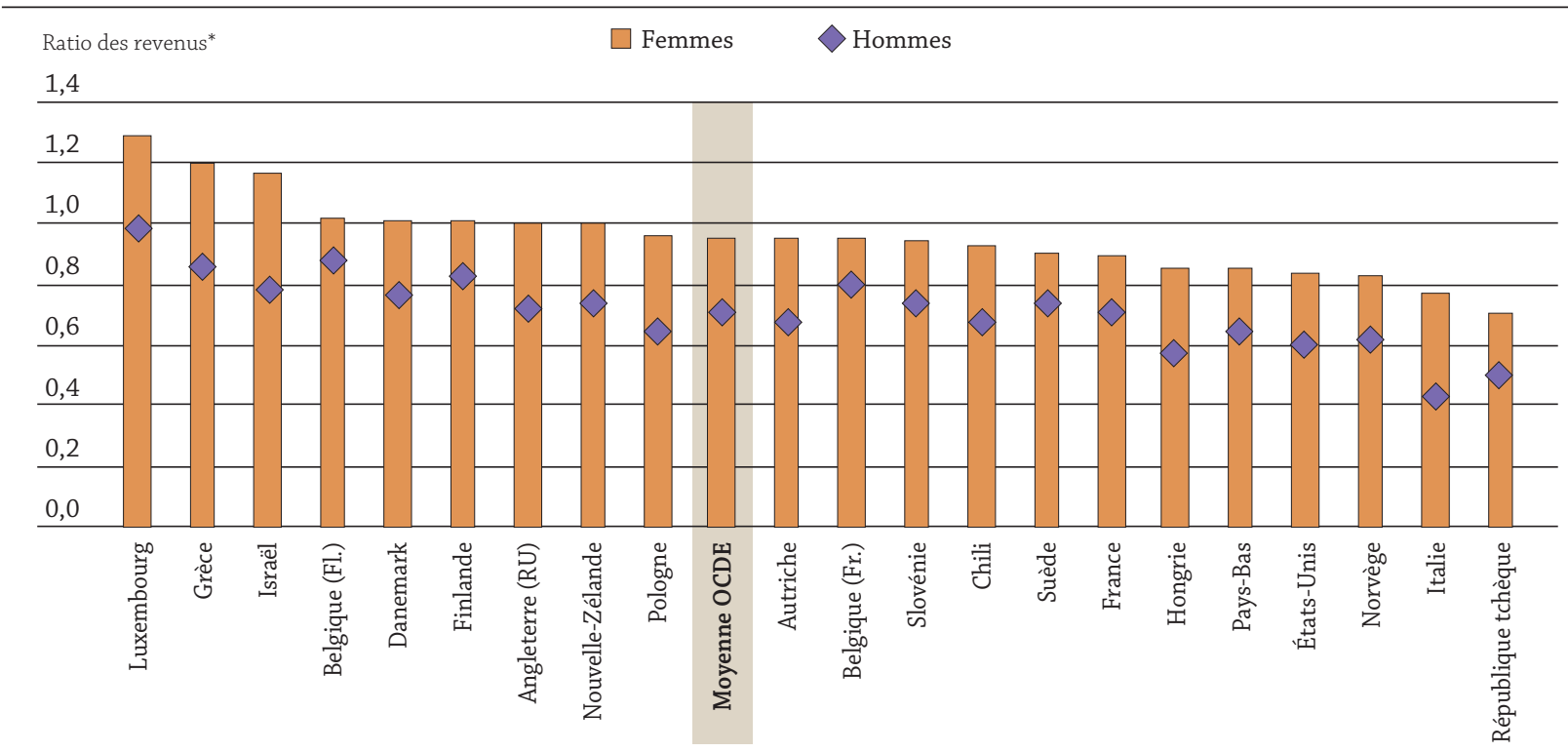

* Le ratio des revenus est calculé en comparant le salaire annuel moyen (primes et allocations comprises) des enseignants (âgés de 25 à 64 ans) des établissements publics aux revenus d'autres actifs du même sexe, diplômés de l'enseignement tertiaire et travaillant à temps plein toute l'année.

Remarque : Seuls sont inclus les pays disposant de données pour l'ensemble des catégories. Pour de plus amples informations, consulter l'annexe 3 de l'édition 2016 de Regards sur l'éducation (www.oecd.org/fr/education/regards-sur-l-education-19991495.htm).

Les pays sont classés par ordre décroissant du ratio entre le salaire des enseignantes et les revenus d'autres actives occupées diplômées de l'enseignement tertiaire. Source : : OCDE (2016), Regards sur l'éducation 2016 : Les indicateurs de l'OCDE, Éditions OCDE, Paris, http://dx.doi.org/10.1787/eag-2016-fr, tableau D3.2C.

\section{Les déséquilibres entre les sexes sont-ils amenés à se creuser dans un avenir proche?}

La pyramide actuelle des âges des enseignants et des enseignantes peut être un bon indicateur des déséquilibres à venir entre les sexes dans la profession. Dans la plupart des pays, le pourcentage le plus élevé de femmes s'observe dans la nouvelle génération d'enseignants, et ce quel que soit le niveau d'enseignement. Ainsi, dans le premier cycle du secondaire, les femmes représentent $70 \%$ des enseignants de moins de 30 ans, contre $65 \%$ des enseignants de 50 ans et plus. Ce constat vaut pour 22 des 35 pays disposant de données, et l'écart entre ces deux groupes d'âge dépasse 15 points de pourcentage dans 8 pays (graphique 4). Le pourcentage plus élevé de femmes parmi les jeunes enseignants suscite des craintes concernant les futurs déséquilibres entre les sexes aux niveaux inférieurs d'enseignement, où les femmes sont déjà majoritaires. En outre, au vu de l'augmentation du pourcentage de femmes diplômées de l'enseignement tertiaire dans le domaine de l'éducation - de $72 \%$ en 2005 à $78 \%$ en 2014, en moyenne, dans les pays de l'OCDE -, tout porte à croire que les déséquilibres entre les sexes sont amenés à s'accentuer dans un avenir proche.

Toutefois, dans l'enseignement tertiaire, où les femmes sont en moyenne minoritaires, l'augmentation du pourcentage de femmes dans la jeune génération de professeurs semble indiquer une amélioration de la parité entre les sexes. En moyenne, dans les pays de l'OCDE, le pourcentage de femmes parmi les professeurs de l'enseignement tertiaire se rapproche de $50 \%$ (soit une répartition égale entre les sexes) chez les plus jeunes - avec $53 \%$ de femmes parmi les professeurs de moins de 30 ans, contre $38 \%$ parmi ceux âgés de 50 ans et plus 2 . Ces indicateurs concordent avec les tendances observées ces dernières décennies dans la répartition du corps enseignant entre les sexes, qui révèlent un creusement progressif de l'écart entre les sexes aux niveaux inférieurs d'enseignement, mais une diminution de cet écart dans l'enseignement tertiaire. En moyenne, dans les pays de I'OCDE, l'augmentation du pourcentage de femmes dans le corps enseignant entre 2005 et 2014 a creusé l'écart entre les sexes dans l'enseignement secondaire (les femmes passant de $59 \%$ à $63 \%$ ) et l'a réduit dans l'enseignement tertiaire (les femmes passant de $38 \%$ à $43 \%$ ).

2. En Chine, en Indonésie et en Turquie, ce constat vaut également pour le premier cycle du secondaire. Les femmes sont largement sous-représentées parmi les enseignants plus âgés, tandis que leur pourcentage se rapproche de $50 \%$ dans la jeune génération d'enseignants. 
Graphique 4. Pourcentage de femmes dans le corps enseignant du premier cycle du secondaire, selon le groupe d'âge (2014)

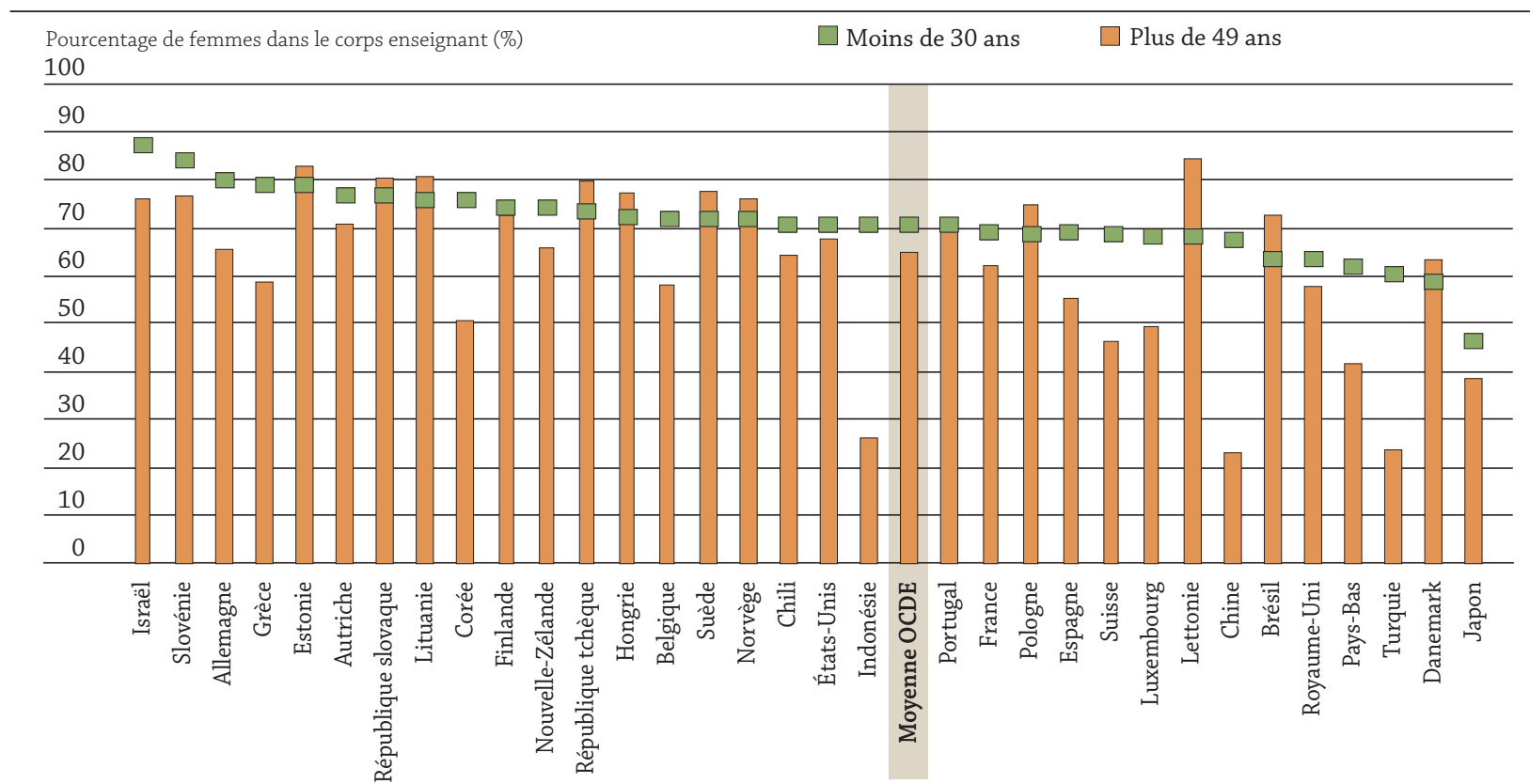

Remarque : Seuls sont inclus les pays disposant de données pour l'ensemble des catégories. Pour de plus amples informations, consulter l'annexe 3 de l'édition 2016 de Regards sur l'éducation (www.oecd.org/fr/education/regards-sur-l-education-19991495.htm).

Les pays sont classés par ordre décroissant du pourcentage de femmes parmi les enseignants de moins de 30 ans.

Source : OCDE (2016), Regards sur l'éducation (base de données).

La persistance des déséquilibres entre les sexes dans la profession enseignante suscite un certain nombre de préoccupations, et des pays comme le Royaume-Uni ont mis en œuvre des mesures encourageant le recrutement d'hommes afin de contrebalancer la « féminisation » croissante de ce métier (OCDE, 2015a). Face à l'ampleur de ce phénomène, et à son accentuation progressive aux niveaux inférieurs d'enseignement, il serait intéressant d'examiner plus avant l'incidence potentielle des écarts entre les sexes dans la profession enseignante, notamment sur les résultats d'apprentissage des élèves ou leurs aspirations professionnelles.

Pour conclure : La profession enseignante est marquée par de forts déséquilibres entre les sexes. Les femmes sont non seulement majoritaires dans le corps enseignant, mais leur répartition entre les différents niveaux d'enseignement et disciplines se caractérise également par sa grande inégalité. Ces disparités entre les sexes suscitent des préoccupations, en particulier face au creusement, ces dix dernières années, de l'écart entre le pourcentage d'hommes et de femmes dans le corps enseignant du primaire et du secondaire. Le pourcentage plus élevé de femmes parmi les enseignants de moins de 30 ans à ces niveaux d'enseignement, ainsi que la prédominance des femmes parmi les diplômés de l'enseignement tertiaire dans le domaine de l'éducation, laissent penser que ce phénomène pourrait s'accentuer dans un avenir proche. Toutefois, des perspectives bien différentes se dessinent dans l'enseignement tertiaire, où la parité entre les sexes s'est améliorée ces dix dernières années, en particulier chez les jeunes professeurs.

\section{Pour tout complément d'information}

- OCDE (2016), Regards sur l'éducation 2016 : Les indicateurs de l'OCDE, Éditions OCDE, Paris, http://dx.doi.org/10.1787/eag-2016-fr.

- OCDE (2015a), " Gender equality ", Trends Shaping Education 2015, Spotlight 7, Éditions OCDE, Paris, www.oecd.org/edu/ceri/ Spotlight7GenderEquality.pdf.

- OCDE (2015b), L'égalité des sexes dans l'éducation: Aptitudes, comportement et confiance, PISA, Éditions OCDE, Paris, http://dx.doi. org/10.1787/9789264230644-fr.

- OCDE (2012), Regards sur l'éducation 2012 : Les indicateurs de l’OCDE, Éditions OCDE, Paris, http://dx.doi.org/10.1787/eag-2012-fr.

\section{Contacter}

Manon Costinot (manon.costinot@oecd.org)

\section{Voir}

www.oecd.org/fr/education/regards-sur-l-education-19991495.htm

Les indicateurs de l'éducation à la loupe (numéros précédents)

PISA à la loupe

L'enseignement à la loupe

\section{Prochain numéro}

Niveau de formation et investissement dans l'éducation dans les pays ibéroaméricains

Crédit photo : ( ) Ghislain \& Marie David de Lossy/Cultura/Getty Images

Ce document est publié sous la responsabilité du Secrétaire général de l'OCDE. Les opinions qui y sont exprimées et les arguments qui y sont employés ne reflètent pas nécessairement les vues officielles des pays membres de l'OCDE.

Ce document, ainsi que les données et cartes qu'il peut comprendre, sont sans préjudice du statut de tout territoire, de la souveraineté s'exerçant sur ce dernier, du tracé des frontières et limites internationales, et du nom de tout territoire, ville ou région.

Les données statistiques concernant Israël sont fournies par et sous la responsabilité des autorités israéliennes compétentes. L'utilisation de ces données par l'OCDE est sans préjudice du statut des hauteurs du Golan, de Jérusalem-Est et des colonies de peuplement israéliennes en Cisjordanie aux termes du droit international.

○ OCDE 2017 Indicateurs de l'éducation à la loupe-février 2017 